\title{
IDEMPOTENT IDEALS AND THE IGUSA-TODOROV FUNCTIONS
}

\author{
A. GATICA, M. LANZILOTTA, M. I. PLATZECK
}

\begin{abstract}
Let $\Lambda$ be an artin algebra and $\mathfrak{A}$ a two-sided idempotent ideal of $\Lambda$, that is, $\mathfrak{A}$ is the trace of a projective $\Lambda$-module $P$ in $\Lambda$. We consider the categories of finitely generated modules over the associated rings $\Lambda / \mathfrak{A}, \Lambda$ and $\Gamma=\operatorname{End}_{\Lambda}(P)^{o p}$ and study the relationship between their homological properties via the Igusa-Todorov functions.
\end{abstract}

\section{INTRODUCTION}

Throughout this paper we assume that $\Lambda$ is an artin algebra and all $\Lambda$ modules are in $\bmod \Lambda$, the category of finitely generated left $\Lambda$-modules.

In 8. Igusa and Todorov introduced two functions $\phi$ and $\psi$ which turned out to be powerful tools to study the finitistic dimension of some classes of algebras. On the other hand, associated to an idempotent ideal $\mathfrak{A}$ of $\Lambda$, there is an exact sequence of categories $\bmod \Lambda / \mathfrak{A} \rightarrow \bmod \Lambda \stackrel{e_{P}}{\longrightarrow} \bmod \Gamma$, where $\mathrm{P}$ is a projective module such that $\mathfrak{A}=\tau_{P} \Lambda$ is the trace of $P$ in $\Lambda, \Gamma=\operatorname{End}_{\Lambda}(P)^{o p}$ and $e_{P}=\operatorname{Hom}_{\Lambda}(P,-)$ is the evaluation functor. In [1] the authors studied the relation between the homological properties of the three categories involved: $\bmod \Lambda / \mathfrak{A}, \bmod \Lambda$ and $\bmod \Gamma$. Our objective in this paper is to study the behaviour of the Igusa-Todorov functions in this situation. For a finitely generated $\Lambda$-module $X$, we will denote $\phi(X)$ by $\phi_{l}^{\Lambda}(X)$, and the supremum of these numbers for $X$ in $\bmod \Lambda$ is the $\phi_{l}$ dimension of $\Lambda$, denoted by $\phi_{l} \operatorname{dim}(\Lambda)$. Additionally, $\operatorname{add} X$ denotes the full subcategory of $\bmod \Lambda$ consisting of summands of finite direct sums of $X$.

First we consider the inclusion of $\bmod \Lambda / \mathfrak{A}$ in $\bmod \Lambda$. To compare the values of the Igusa-Todorov functions in a $\Lambda / \mathfrak{A}$-module $X$ in both categories we need the further assumption that the idempotent ideal $\mathfrak{A}$ is a strong idempotent ideal, in the sense defined in [1]. We recall that the ideal $\mathfrak{A}$ is a strong idempotent ideal if the morphism $\operatorname{Ext}_{\Lambda / \mathfrak{A}}^{i}(X, Y) \rightarrow \operatorname{Ext}_{\Lambda}^{i}(X, Y)$ induced by

\footnotetext{
Key words and phrases. Idempotent ideals, Igusa-Todorov functions, homological dimensions, artin algebras.

2010 Mathematics Subject Classification. 16E10, 16G10.

The authors thank the financial support received from Universidad de la República, Montevideo, Uruguay, from Universidad Nacional del Sur, Bahía Blanca, Argentina and from CONICET, Argentina.
} 
the canonical isomorphism $\operatorname{Hom}_{\Lambda / \mathfrak{A}}(X, Y) \rightarrow \operatorname{Hom}_{\Lambda}(X, Y)$ is an isomorphism for all $i \geq 0$ and all $X, Y$ in $\bmod \Lambda / \mathfrak{A}$. We prove that $\phi_{l}^{\Lambda / \mathfrak{A}}(X) \leq \phi_{l}^{\Lambda}(X)$ for all $X \in \bmod \Lambda / \mathfrak{A}$, whenever $\mathfrak{A}$ is a strong idempotent ideal of finite projective dimension. Thus in this case the $\phi_{l}$ dimension of $\Lambda / \mathfrak{A}$ is bounded by the $\phi_{l}$ dimension of $\Lambda$.

In order to compare the behaviour of the Igusa-Todorov functions under the functor $\bmod \Lambda \stackrel{e_{P}}{\longrightarrow} \bmod \Gamma$, we recall that $e_{P}$ induces an equivalence between the full subcategory of $\bmod \Lambda$ consisting of the $\Lambda$-modules $X$ having a presentation in $\operatorname{add} P$, and $\bmod \Gamma$. We prove that both functions $\phi$ and $\psi$ are preserved under $e_{P}$ for modules having a resolution in $\operatorname{add} P$. As a consequence we obtain that when all $\Lambda$-modules with a presentation in $\operatorname{add} P$ have also a resolution in $\operatorname{add} P$, then $\phi_{l} \operatorname{dim} \Gamma \leq \phi_{l} \operatorname{dim} \Lambda$ and $\psi_{l} \operatorname{dim} \Gamma \leq \psi_{l} \operatorname{dim} \Lambda$ (Theorem 4.7).

Then we obtain information about the $\phi$ dimension of $\Lambda$ from the $\phi$ dimensions of the algebras $\Lambda / \mathfrak{A}$ and $\Gamma$. We prove several inequalities, which are interesting when either the global dimension of $\Lambda / \mathfrak{A}$ or the global dimension of $\Gamma$ are finite.

To prove these results we use, in one hand, the characterization of the Igusa-Todorov function $\phi$ in terms of the bifunctor $\operatorname{Ext}(-,-)$ given in [5]. On the other hand, the full subcategory $\mathbb{T}$ of $\bmod \Lambda$ introduced in [1] consisting of the modules $T$ such that $\operatorname{Ext}_{\Lambda}^{i}(\Lambda / \mathfrak{A}, T)=0$ for all $i \geq 1$, is very useful for our purposes. Consider the full subcategories $\mathbb{P}_{0}$ and $\mathbb{P}_{\infty}$ of $\bmod \Lambda$, where $\mathbb{P}_{0}$ consists of the modules whose projective cover is in $\operatorname{add} P$, and $\mathbb{P}_{\infty}$ of those having a projective resolution in $\operatorname{add} P$. We use the fact, proven in section 3 , that $\left(\mathbb{P}_{0}, \bmod \Lambda / \mathfrak{A}\right)$ is a torsion pair in $\bmod \Lambda$ whose properties are inherited by the pair $\left(\mathbb{P}_{\infty}, \bmod \Lambda / \mathfrak{A}\right)$ in the category $\tilde{\mathbb{T}}$ dual of $\mathbb{T}$.

\section{Preliminaries}

Let $\Lambda$ be an artin algebra, $M$ and $N$ in $\bmod \Lambda$. We denote by $\tau_{M} N$ the trace of $M$ in $N$, that is, the submodule of $N$ generated by the homomorphic images of maps from $M$ to $N$. Moreover, $P_{0}(M), I_{0}(M)$ denote the projective cover and injective envelope of $M$, and $\Omega^{n}(M), \Omega^{-n}(M)$ the $n^{\text {th }}$ syzygy and the $n^{\text {th }}$ cosyzygy of $M$, respectively. Finally, $\operatorname{pd} M$ denotes the projective dimension of $\mathrm{M}$ and gld $\Lambda$ stands for the global dimension of $\Lambda$.

We start by recalling some definitions and results from [1] which will be used throughout the paper. Let $\mathfrak{A}$ be an idempotent ideal of $\Lambda, P_{0}$ the projective cover of $\mathfrak{A}$, and $P=\Lambda e$ where $e$ is an idempotent element of $\Lambda$ such that $\operatorname{add} P=\operatorname{add} P_{0}$. Then $\mathfrak{A}=\Lambda e \Lambda=\tau_{P} \Lambda$ is the trace of $P$ in $\Lambda, \bmod \Lambda / \mathfrak{A}$ is a Serre subcategory of $\bmod \Lambda$ and this inclusion induces an exact sequence of categories $\bmod \Lambda / \mathfrak{A} \rightarrow \bmod \Lambda \stackrel{e_{P}}{\longrightarrow} \bmod \Gamma$, where $\Gamma=\operatorname{End}_{\Lambda}(P)^{o p}$ and $e_{P}=$ $\operatorname{Hom}_{\Lambda}(P,-)$ is the evaluation functor. 
Let $P / r P \simeq S_{1} \oplus \cdots \oplus S_{r}$, with $S_{i}$ simple for all $i$, so that $P=P_{0}\left(S_{1} \oplus \cdots \oplus\right.$ $\left.S_{r}\right)$, and let $I=I_{0}\left(S_{1} \oplus \cdots \oplus S_{r}\right)$. To compare the homological properties of $\bmod \Lambda$ and $\bmod \Gamma$, full subcategories $\mathbb{P}_{k}$ and $\mathbb{I}_{k}$ were introduced in 1 for any $k \geq 0$. These subcategories will be useful for our purposes, and are defined as follows: $\mathbb{P}_{k}$ is the full subcategory of $\bmod \Lambda$ consisting of the $\Lambda$-modules $X$ having a projective resolution $\cdots \rightarrow P_{1} \rightarrow P_{0} \rightarrow X \rightarrow 0$ with $P_{i}$ in add $P$ for $0 \leq i \leq k$. The full subcategory $\mathbb{I}_{k}$ is defined dually.

Then $\operatorname{Hom}_{\Lambda}(P,-)$ induces equivalences $\mathbb{P}_{1} \rightarrow \bmod \Gamma$ and $\mathbb{I}_{1} \rightarrow \bmod \Gamma$. Moreover, the morphism of connected sequences of functors $\operatorname{Ext}_{\Lambda}^{i}(X, Y) \rightarrow$ $\operatorname{Ext}_{\Gamma}^{i}((P, X),(P, Y))$ induced by $\operatorname{Hom}_{\Lambda}(P,-)$ is an isomorphism for $i=1, \cdots, k$, whenever $X \in \mathbb{P}_{(k+1)}$ or $Y \in \mathbb{I}_{(k+1)}$ (Theorem 3.2, [1]).

We next turn our attention to the definition of the Igusa-Todorov functions, defined in [8. Let $K_{0}$ denote the abelian group generated by all symbols $[M]$, where $M$ in $\bmod \Lambda$, modulo the relations a) $[C]=[A]+[B]$ if $C \simeq A \oplus B$ and b) $[P]=0$ if $P$ is projective. That is, $K_{0}$ is the free abelian group generated by the isomorphism classes of indecomposable finitely generated nonprojective $\Lambda$-modules. Let $\Omega: K_{0} \rightarrow K_{0}$ denote the group homomorphism induced by the syzygy, that is, $\Omega([M]):=[\Omega(M)]$, and let $<\operatorname{add} M>$ be the subgroup of $K_{0}$ generated by the indecomposable sumands of $M$. When we apply the homomorphism $\Omega$ to this subgroup the rank does not increase: rank $\Omega(<\operatorname{add} M>) \leq \operatorname{rank}<\operatorname{add} M>$, and there is then an integer $n$ such that $\Omega: \Omega^{s}(<\operatorname{add} M>) \rightarrow \Omega^{s+1}(<\operatorname{add} M>)$ is an isomorphism for all $s \geq n$. Then the Igusa-Todorov functions $\phi$ and $\psi$ are defined as follows: $\phi(M)$ is the smallest non-negative integer $n$ with this property, and $\psi(M):=\phi(M)+$ $\sup \left\{\operatorname{pd} X \mid X\right.$ is a direct summand of $\Omega^{\phi(M)}(M)$ with $\left.\operatorname{pd} X<\infty\right\}$. Since we will need also the dual notions, we will denote the Igusa-Todorov functions $\phi$ and $\psi$ by $\phi_{l}$ and $\psi_{l}$, respectively. Using the cozyzygy we can define $\phi_{r}(M)$ and $\psi_{r}(M)$ in an analogous way. Then $\phi_{r}(M)=\phi_{l}(D M)$ and $\psi_{r}(M)=\psi_{l}(D M)$, for any $M$ in $\bmod \Lambda$.

Let $\phi_{l} \operatorname{dim} \Lambda=\sup \left\{\phi_{l}(M) \mid M\right.$ in $\left.\bmod \Lambda\right\}$ and $\psi_{l} \operatorname{dim} \Lambda=\sup \left\{\psi_{l}(M) \mid M\right.$ in $\bmod \Lambda\}$. Moreover, for a subcategory $\mathcal{X}$ of $\bmod \Lambda$ we indicate by $\phi_{l} \operatorname{dim} \mathcal{X}$ and $\psi_{l} \operatorname{dim} \mathcal{X}$ the supremum of the sets $\left\{\phi_{l}(X) \mid X\right.$ in $\left.\mathcal{X}\right\}$ and $\left\{\psi_{l}(X) \mid X\right.$ in $\left.\mathcal{X}\right\}$, respectively. Analogous notions are defined for $\phi_{r}$ and $\psi_{r}$.

We will also need the characterization of the function $\phi$ in terms of the bifunctor $\operatorname{Ext}_{\Lambda}^{i}(-,-)$ given in $[5$. We recall first that a pair $(X, Y)$ of objects in $\operatorname{add} M$ is called $d$-division of $M$ if the following three conditions hold:

(a) $\operatorname{add}(X) \cap \operatorname{add}(Y)=0$

(b) $\operatorname{Ext}_{\Lambda}^{d}(X,-) \not \operatorname{Ext}_{\Lambda}^{d}(Y,-)$ in $\bmod \Lambda$

(c) $\operatorname{Ext}_{\Lambda}^{d+1}(X,-) \simeq \operatorname{Ext}_{\Lambda}^{d+1}(Y,-)$ in $\bmod \Lambda$.

Dually, a pair $(X, Y)$ of objects in $\operatorname{add} M$ is called d-injective division of $M$ if (a) and the following two conditions hold:

(b') $\operatorname{Ext}_{\Lambda}^{d}(-, X) \not \operatorname{Ext}_{\Lambda}^{d}(-, Y)$ in $\bmod \Lambda$ 
(c') $\operatorname{Ext}_{\Lambda}^{d+1}(-, X) \simeq \operatorname{Ext}_{\Lambda}^{d+1}(-, Y)$ in $\bmod \Lambda$.

Then $\phi_{l}(M)=\max (\{d \in \mathbb{N}$ :there is a d-division of $M\} \cup\{0\})([5$, Theorem 3.6), and $\phi_{r}(M)=\max (\{d \in \mathbb{N}$ :there is a d-injective division of $M\} \cup\{0\})$.

\section{TORSION THEORIES ASSOCIATED TO AN IDEMPOTENT IDEAL}

It is interesting to notice that the idempotent ideal $\mathfrak{A}$ determines two torsion pairs $\left(\bmod \Lambda / \mathfrak{A}, \mathbb{I}_{0}\right)$ and $\left(\mathbb{P}_{0}, \bmod \Lambda / \mathfrak{A}\right)$ in $\bmod \Lambda$, in the sense defined by Dickson in 4], as we state in the following proposition.

Proposition 3.1. Let $\mathfrak{A}$ be an idempotent ideal of $\Lambda, \mathfrak{A}=\tau_{P} \Lambda$, where $P$ is a projective $\Lambda$-module. Then

(a) $\left(\bmod \Lambda / \mathfrak{A}, \mathbb{I}_{0}\right)$ is a torsion pair in $\bmod \Lambda$.

(b) $\left(\mathbb{P}_{0}, \bmod \Lambda / \mathfrak{A}\right)$ is a torsion pair in $\bmod \Lambda$.

Proof. (a) To prove this we observe that $\mathbb{I}_{0}$ consists of the modules with socle in $\operatorname{add}\left(S_{1} \oplus \cdots \oplus S_{r}\right)$, and that a $\Lambda$-module $M$ is in $\bmod \Lambda / \mathfrak{A}$ if and only if $S_{1}, \cdots, S_{r}$ are not composition factors of $M$. Then $\operatorname{Hom}_{\Lambda}(M, Y)=0$ for $M \in \bmod \Lambda / \mathfrak{A}$ and $Y \in \mathbb{I}_{0}$. Moreover, if $\operatorname{Hom}_{\Lambda}(M, Y)=0$ for all $Y \in \mathbb{I}_{0}$, then in particular $\operatorname{Hom}_{\Lambda}\left(M, I_{0}\left(S_{1} \oplus \cdots \oplus S_{r}\right)\right)=0$, so that $S_{1}, \cdots, S_{r}$ are not composition factors of $\mathrm{M}$, and $M$ is thus a $\Lambda / \mathfrak{A}$-module. Finally, suppose that $\operatorname{Hom}_{\Lambda}(M, Y)=0$ for each $M \in \bmod \Lambda / \mathfrak{A}$. Then $\operatorname{Hom}_{\Lambda}(S, Y)=0$ for any simple $S$ not isomorphic to $S_{1}, \cdots, S_{r}$. Thus the only simples in the socle of $Y$ are amongst $S_{1}, \cdots, S_{r}$, and therefore $Y \in \mathbb{I}_{0}$. This shows that $\left(\bmod \Lambda / \mathfrak{A}, \mathbb{I}_{0}\right)$ is a torsion pair in $\bmod \Lambda$.

The statement (b) follows by duality.

In the sequel we will consider the full subcategory $\mathbb{T}$ of $\bmod \Lambda$ introduced and studied in section 5 of [1], consisting of the modules $T$ such that the group $\operatorname{Ext}_{\Lambda}^{i}(\Lambda / \mathfrak{A}, T)=0$ for all $i \geq 1$. Dually, we define the subcategory $\tilde{\mathbb{T}}=D\left(\mathbb{T}_{\Lambda}\right)$ consisting of the $\Lambda$-modules $X$ such that $\operatorname{Ext}_{\Lambda}^{i}\left(X, D\left(\Lambda / \mathfrak{A}_{\Lambda}\right)\right)=0$ for all $i \geq 1$.

The notion of torsion pairs in abelian categories defined by Dickson was extended to pretriangulated categories by Beligianis and Reiten (see [2], Ch. II, Definition 3.1). Additive categories with kernels and cokernels are examples of pretriangulated categories, as shown in section 1, Example 2 of the same paper, and in this case torsion pairs are defined as follows.

Definition 3.2. ([2] A pair of subcategories $(\mathcal{X}, \mathcal{Y})$ in an additive category $\mathcal{C}$ with kernels and cokernels and closed under isomorphisms is a torsion pair if the following conditions hold:

T1) $\operatorname{Hom}_{\mathcal{C}}(X, Y)=0$ for all $X \in \mathcal{X}, Y \in \mathcal{Y}$

T2) For every $C \in \mathcal{C}$ there is an exact sequence $0 \rightarrow X_{C} \rightarrow C \rightarrow Y_{C} \rightarrow 0$ with $X_{C} \in \mathcal{X}, Y_{C} \in \mathcal{Y}$ (glueing sequence). 
We observe that for the torsion pairs $\left(\bmod \Lambda / \mathfrak{A}, \mathbb{I}_{0}\right)$ and $\left(\mathbb{P}_{0}, \bmod \Lambda / \mathfrak{A}\right)$ above considered the glueing sequences for a module $X$ in $\bmod \Lambda$ are $0 \rightarrow$ $\tau_{\Lambda / \mathfrak{A}} X \rightarrow X \rightarrow X / \tau_{\Lambda / \mathfrak{A}} X \rightarrow 0$ and $0 \rightarrow \tau_{\mathfrak{A}} X \rightarrow X \rightarrow X / \tau_{\mathfrak{A}} X \rightarrow 0$ respectively.

We now turn our attention to the subcategories $\mathbb{T}$ and $\tilde{\mathbb{T}}$ of $\bmod \Lambda$, and study the restriction of these torsion pairs to $\mathbb{T}$ and $\tilde{\mathbb{T}}$ respectively, under the assumption that the ideal $\mathfrak{A}$ is strong idempotent.

Proposition 3.3. Let $\mathfrak{A}$ be a strong idempotent ideal of $\Lambda$. Then

(a) $\mathbb{I}_{\infty}=\mathbb{I}_{0} \cap \mathbb{T}$, and the pair $\left(\bmod \Lambda / \mathfrak{A}, I_{\infty}\right)$ of subcategories of $\mathbb{T}$ satisfies conditions T1) and T2) of Definition 3.2 of torsion pair. Moreover, for $T$ in $\mathbb{T}$ the glueing sequence is $0 \rightarrow \tau_{\Lambda / \mathfrak{A}} T \rightarrow T \rightarrow T / \tau_{\Lambda / \mathfrak{A}} T \rightarrow 0$.

(b) $\mathbb{P}_{\infty}=\mathbb{P}_{0} \cap \tilde{\mathbb{T}}$, and the pair $\left(\bmod \Lambda / \mathfrak{A}, P_{\infty}\right)$ of subcategories of $\tilde{\mathbb{T}}$ satisfies conditions T1) and T2) of Definition 3.2 of torsion pair. Moreover, for $T \in \tilde{\mathbb{T}}$ the glueing sequence is $0 \rightarrow \tau_{\mathfrak{A}} T \rightarrow T \rightarrow T / \tau_{\mathfrak{A}} T \rightarrow 0$.

Proof. Since $\mathbb{I}_{\infty} \subseteq \mathbb{I}_{0}$ then condition T1) in the definition of torsion pair holds. Assume now that $\mathfrak{A}$ is a strong idempotent ideal. Then $\bmod \Lambda / \mathfrak{A} \subset \mathbb{T}$. In fact, if $X \in \bmod \Lambda / \mathfrak{A}$ then $\operatorname{Ext}_{\Lambda}^{i}(\Lambda / \mathfrak{A}, X) \cong \operatorname{Ext}_{\Lambda / \mathfrak{A}}^{i}(\Lambda / \mathfrak{A}, X)=0$ for all $i \geq 0$, so $X \in \mathbb{T}$. On the other hand, we know that a module $Y$ is in $\mathbb{I}_{\infty}$ if and only if $\operatorname{Ext}_{\Lambda}^{i}(\Lambda / \mathfrak{A}, Y)=0$ for all $i \geq 0$, by [1], Proposition 2.6. Thus, it follows from the definition of $\mathbb{T}$ that $\mathbb{I}_{\infty}=\mathbb{I}_{0} \cap \mathbb{T}$.

Therefore, for $T \in \mathbb{T}$ the exact sequence $0 \rightarrow \tau_{\Lambda / \mathfrak{A}} T \rightarrow T \rightarrow T / \tau_{\Lambda / \mathfrak{A}} T \rightarrow 0$ has $\tau_{\Lambda / \mathfrak{A}} T$ in $\bmod \Lambda / \mathfrak{A}$ and $T / \tau_{\Lambda / \mathfrak{A}} T$ in $\mathbb{I}_{\infty}$ and is then a glueing sequence for $T$. This proves condition T2) and ends the proof of (a). The proof of (b) is similar.

Remark 3.4. Though we do not know wether the subcategories $\mathbb{T}$ and $\tilde{\mathbb{T}}$ have kernels and cokernels, we observe that the category $\mathbb{T}$ is not in general abelian as the following simple example shows. Let $\Lambda$ be the path algebra of the quiver $1 \rightarrow 2$, and $P=S_{2}$, the simple projective module associated to the vertex 2. Then, $\mathfrak{A}=\tau_{P} \Lambda \simeq S_{2} \oplus S_{2}$ is a projective $\Lambda$ module and therefore it is a strong idempotent ideal. Moreover, $\mathbb{T}=\operatorname{add}\left\{\begin{array}{l}S_{1} \\ S_{2}\end{array}, S_{1}\right\}$ and $\Lambda / \mathfrak{A} \simeq S_{1}$. Consider the exact sequence $0 \rightarrow S_{2} \rightarrow \underset{S_{2}}{S_{1}} \stackrel{f}{\rightarrow} S_{1} \rightarrow 0$. Then $f$ is a map in $\mathbb{T}$, and $\operatorname{Ker}_{\mathbb{T}}(f)=0$, because there are no nonzero maps from objects in $\mathbb{T}$ to $S_{2}$. Thus $\operatorname{Coker}_{\mathbb{T}}\left(\operatorname{Ker}_{\mathbb{T}}(f)\right)=\operatorname{Coker}_{\mathbb{T}}\left(0 \rightarrow \begin{array}{l}S_{1} \\ S_{2}\end{array}\right)=\left(\begin{array}{c}S_{1} \\ S_{2}\end{array} \stackrel{i d}{\longrightarrow} \begin{array}{l}S_{1} \\ S_{2}\end{array}\right)$. However, $\operatorname{Ker}_{\mathbb{T}}\left(\operatorname{Coker}_{\mathbb{T}}(f)\right)=\operatorname{Ker}_{\mathbb{T}}\left(S_{1} \rightarrow 0\right)=\left(S_{1} \stackrel{i d}{\rightarrow} S_{1}\right)$.

In connection with the torsion pairs and subcategories above considered we prove two technical lemmas which will be useful throughout the paper.

Lemma 3.5. Let $\mathfrak{A}$ be an idempotent ideal. Then 
(a) $\operatorname{Hom}_{\Lambda}(P, X) \simeq \operatorname{Hom}_{\Lambda}\left(P, X / \tau_{\Lambda / \mathfrak{A}} X\right)$ for all $X \in \bmod \Lambda$.

(b) $\left.\left.\operatorname{Ext}_{\Lambda}^{j}(-, X)\right|_{\mathbb{P}_{\infty}} \simeq \operatorname{Ext}_{\Lambda}^{j}\left(-, X / \tau_{\Lambda / \mathfrak{A}} X\right)\right|_{\mathbb{P}_{\infty}}$ for all $j \geq 0$ and $X \in$ $\bmod \Lambda$.

(c) $\operatorname{Hom}_{\Lambda}(X, I) \simeq \operatorname{Hom}_{\Lambda}\left(\tau_{\mathfrak{A}} X, I\right)$ for all $X \in \bmod \Lambda$.

(d) $\left.\left.\operatorname{Ext}_{\Lambda}^{j}(X,-)\right|_{\mathbb{I}_{\infty}} \simeq \operatorname{Ext}_{\Lambda}^{j}\left(\tau_{\mathfrak{A}} X,-\right)\right|_{\mathbb{I}_{\infty}}$ for all $j \geq 0$ and $X \in \bmod \Lambda$.

Proof. (a) The result follows directly by applying the exact functor $\operatorname{Hom}_{\Lambda}(P,-)$ to the exact sequence $0 \rightarrow \tau_{\Lambda / \mathfrak{A}} X \rightarrow X \rightarrow X / \tau_{\Lambda / \mathfrak{A}} X \rightarrow 0$.

(b) We recall from [1], Theorem $3.2 \mathrm{c})$, that there is a functorial isomorphism $\left.\left.\operatorname{Ext}_{\Lambda}^{j}(-, Y)\right|_{\mathbb{P}_{\infty}} \simeq \operatorname{Ext}_{\Gamma}^{j}((P,-),(P, Y))\right|_{\mathbb{P}_{\infty}}$ for all $Y$ in $\bmod \Lambda$. The result follows now using (a).

By duality we obtain the statements (c) and (d).

When we further assume that the ideal $\mathfrak{A}$ is strong idempotent we get the following result.

Lemma 3.6. Let $\mathfrak{A}$ be a strong idempotent ideal. Then

(a) $\left.\operatorname{Ext}_{\Lambda}^{j}\left(-, X / \tau_{\Lambda / \mathfrak{A}} X\right)\right|_{\bmod \Lambda / \mathfrak{A}}=0$ for all $X \in \mathbb{T}$ and $j \geq 1$.

(b) $\operatorname{Ext}_{\Lambda}^{j}\left(-, X_{1}\right) \simeq \operatorname{Ext}_{\Lambda}^{j}\left(-, X_{2}\right)$ with $j \geq 0$ implies

$\left.\left.\operatorname{Ext}_{\Lambda}^{j}\left(-, X_{1} / \tau_{\Lambda / \mathfrak{A}} X_{1}\right)\right|_{\tilde{\mathbb{T}}} \simeq \operatorname{Ext}_{\Lambda}^{j}\left(-, X_{2} / \tau_{\Lambda / \mathfrak{A}} X_{2}\right)\right|_{\tilde{\mathbb{T}}}$ for all $X_{1}, X_{2} \in \mathbb{T}$.

(c) $\operatorname{Ext}_{\Lambda}^{j}\left(\tau_{\mathfrak{A}} X,-\right)=0$ for all $X \in \tilde{\mathbb{T}}$ and $j \geq 1$.

(d) $\operatorname{Ext}_{\Lambda}^{j}\left(X_{1},-\right) \simeq \operatorname{Ext}_{\Lambda}^{j}\left(X_{2},-\right)$ with $j \geq 0$ implies

$\left.\left.\operatorname{Ext}_{\Lambda}^{j}\left(\tau_{\mathfrak{A}} X_{1},-\right)\right|_{\mathbb{T}} \simeq \operatorname{Ext}_{\Lambda}^{j}\left(\tau_{\mathfrak{A}} X_{2},-\right)\right|_{\mathbb{T}}$ for all $X_{1}, X_{2} \in \tilde{\mathbb{T}}$.

Proof. (a) Let $X$ in $\mathbb{T}$ and $Z$ in $\bmod \Lambda / \mathfrak{A}$. Then $X / \tau_{\Lambda / \mathfrak{A}} X \in \mathbb{I}_{\infty}$ and using (d) of the previous lemma we conclude that $\operatorname{Ext}_{\Lambda}^{j}\left(Z, X / \tau_{\Lambda / \mathfrak{A}} X\right) \simeq \operatorname{Ext}_{\Lambda}^{j}\left(\tau_{\mathfrak{A}} Z, X / \tau_{\Lambda / \mathfrak{A}} X\right)=$ 0 , since $\tau_{\mathfrak{A}} Z=0$ because $Z$ is a $\Lambda / \mathfrak{A}$-module.

(b) Let $X_{1}, X_{2} \in \mathbb{T}$ be such that $\operatorname{Ext}_{\Lambda}^{j}\left(-, X_{1}\right) \simeq \operatorname{Ext}_{\Lambda}^{j}\left(-, X_{2}\right)$. So $L_{1}=$ $X_{1} / \tau_{\Lambda / \mathfrak{A}} X_{1}, L_{2}=X_{2} / \tau_{\Lambda / \mathfrak{A}} X_{2} \in \mathbb{I}_{\infty}$. Then we obtain from (a) that $\left.\operatorname{Ext}_{\Lambda}^{j}\left(-, L_{i}\right)\right|_{\bmod \Lambda / \mathfrak{A}}=$ 0 for $i=1,2$ and for all $j \geq 1$.

Let $Z \in \tilde{\mathbb{T}}$. Applying the functor $\operatorname{Hom}_{\Lambda}\left(-, L_{i}\right)$ to the glueing sequence

$$
0 \longrightarrow \tau_{\mathfrak{A}} Z \longrightarrow Z \longrightarrow Z / \tau_{\mathfrak{A}} Z \longrightarrow 0
$$

the corresponding long exact sequence yields isomorphisms

$$
\operatorname{Ext}_{\Lambda}^{j}\left(Z, L_{i}\right) \simeq \operatorname{Ext}_{\Lambda}^{j}\left(\tau_{\mathfrak{A}} Z, L_{i}\right)
$$

for $i=1,2$ and $j \geq 1$.

On the other hand, by (b) of the previous lemma we know that $\operatorname{Ext}_{\Lambda}^{j}\left(\tau_{\mathfrak{A}} Z, X_{i}\right) \simeq$ $\operatorname{Ext}_{\Lambda}^{j}\left(\tau_{\mathfrak{A}} Z, L_{i}\right)$ for $i=1,2, j \geq 1$ because $\mathfrak{A}$ is a strong idempotent ideal, so $Z$

in $\tilde{\mathbb{T}}$ implies that $Z$ is a $\Lambda / \mathfrak{A}$-module. Then, in the commutative diagram 


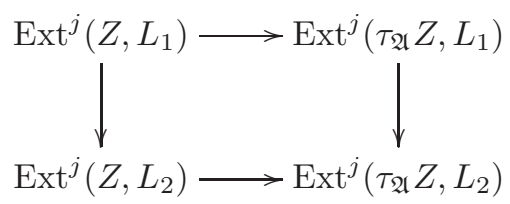

the horizontal arrows and the right vertical arrow are isomorphisms when $j \geq 1$. This proves the left vertical arrow is also an isomorphism in this case, as desired.

Finally, statements (c) and (d) follow from (a) and (b) by duality.

\section{MAIn RESUlts}

Next we turn our attention to the functions $\phi$ and $\psi$ defined by Igusa and Todorov. We are going to use the characterization of these functions in terms of the functor Ext given in [5]. We start with two lemmas comparing the behaviour of this functor in $\bmod \Lambda$ and $\operatorname{in} \bmod \Lambda / \mathfrak{A}$.

Lemma 4.1. Let $\mathfrak{A}$ be an idempotent ideal such that $\operatorname{pd}\left({ }_{\Lambda} \Lambda / \mathfrak{A}\right)=r<\infty$. Let $X_{1}, X_{2} \in \bmod \Lambda / \mathfrak{A}$ and $t \geq 1$. Then $\operatorname{Ext}_{\Lambda / \mathfrak{A}}^{t}\left(X_{1},-\right) \simeq \operatorname{Ext}_{\Lambda / \mathfrak{A}}^{t}\left(X_{2},-\right)$ implies $\operatorname{Ext}_{\Lambda}^{t+r}\left(X_{1},-\right) \simeq \operatorname{Ext}_{\Lambda}^{t+r}\left(X_{2},-\right)$.

Proof. Note first that $\operatorname{Ext}_{\Lambda}^{t+r}\left(X_{j},-\right) \simeq \operatorname{Ext}_{\Lambda}^{t}\left(X_{j}, \Omega^{-r}(-)\right)$, for $j=1,2$. Since $\Omega^{-r}(\bmod \Lambda) \subset \mathbb{T}$, from Lemma 5.5, 1], using Proposition 1.1, [1, it follows that $\operatorname{Ext}_{\Lambda / \mathfrak{A}}^{i}\left(X_{j}, \tau_{\Lambda / \mathfrak{A}}\left(\Omega^{-r}(-)\right)\right) \simeq \operatorname{Ext}_{\Lambda}^{i}\left(X_{j}, \Omega^{-r}(-)\right)$, for all $1 \leq i$, and $j=$ 1,2 .

Now $\operatorname{Ext}_{\Lambda}^{t+r}\left(X_{1},-\right) \simeq \operatorname{Ext}_{\Lambda}^{t}\left(X_{1}, \Omega^{-r}(-)\right) \simeq \operatorname{Ext}_{\Lambda / \mathfrak{A}}^{t}\left(X_{1}, \tau_{\Lambda / \mathfrak{A}}\left(\Omega^{-r}(-)\right)\right) \simeq$ $\operatorname{Ext}_{\Lambda / \mathfrak{A}}^{t}\left(X_{2}, \tau_{\Lambda / \mathfrak{A}}\left(\Omega^{-r}(-)\right)\right) \simeq \operatorname{Ext}_{\Lambda}^{t+r}\left(X_{2},-\right)$

Lemma 4.2. Let $\mathfrak{A}$ be an idempotent ideal such that $\operatorname{pd}\left(\Lambda / \mathfrak{A}_{\Lambda}\right)=r<\infty$. Let $X_{1}, X_{2} \in \bmod \Lambda / \mathfrak{A}$ and $t \geq 1$. Then $\operatorname{Ext}_{\Lambda / \mathfrak{A}}^{t}\left(-, X_{1}\right) \simeq \operatorname{Ext}_{\Lambda / \mathfrak{A}}^{t}\left(-, X_{2}\right)$ implies $\operatorname{Ext}_{\Lambda}^{t+r}\left(-, X_{1}\right) \simeq \operatorname{Ext}_{\Lambda}^{t+r}\left(-, X_{2}\right)$.

Proof. It follows from Lemma 4.1 by duality, using that $\operatorname{Ext}_{\Lambda / \mathfrak{A}}^{j}(-, Y) \simeq$ $\operatorname{Ext}_{\Lambda / \mathfrak{A}^{\circ p}}^{j}(D Y, D(-))$.

We prove next that when the ideal $\mathfrak{A}$ is a strong idempotent ideal of finite projective dimension then the $\phi$ dimension of the factor algebra $\Lambda / \mathfrak{A}$ is bounded by the $\phi$ dimension of $\Lambda$.

Theorem 4.3. Let $\mathfrak{A}$ be a strong idempotent ideal of $\Lambda$ such that $\operatorname{pd}(\Lambda \Lambda / \mathfrak{A})=$ $r<\infty$. Then

(a) $\phi_{l}^{\Lambda / \mathfrak{A}}(X) \leq \phi_{l}^{\Lambda}(X)$ for all $X \in \bmod \Lambda / \mathfrak{A}$.

(b) $\phi_{l} \operatorname{dim} \Lambda / \mathfrak{A} \leq \phi_{l} \operatorname{dim} \Lambda$. 
Proof. Let $d$ a positive integer and assume that $X=X_{1} \oplus X_{2}$ is a $d$-division of the $\Lambda / \mathfrak{A}$-module $X$. This is, $\operatorname{Ext}_{\Lambda / \mathfrak{A}}^{d}\left(X_{1},-\right) \neq \operatorname{Ext}_{\Lambda / \mathfrak{A}}^{d}\left(X_{2},-\right)$ and $\operatorname{Ext}_{\Lambda / \mathfrak{A}}^{d+1}\left(X_{1},-\right) \simeq$ $\operatorname{Ext}_{\Lambda / \mathfrak{A}}^{d+1}\left(X_{2},-\right)$. Since $\mathfrak{A}$ is a strong idempotent ideal then $\operatorname{Ext}_{\Lambda}^{d}\left(X_{1},-\right) \not$ $\operatorname{Ext}_{\Lambda}^{d}\left(X_{2},-\right)$. On the other hand, we know that $\phi_{l}^{\Lambda}(X)=\max (\{d \in \mathbb{N}$ : there is a $d$-division of $X$ in $\bmod \Lambda\} \cup\{0\})$, by Theorem 3.6 in [5. Thus to prove that $\phi_{l}^{\Lambda}(X) \geq d$, it is enough to find $l>d$ such that $\operatorname{Ext}_{\Lambda}^{l}\left(X_{1},-\right) \simeq \operatorname{Ext}_{\Lambda}^{l}\left(X_{2},-\right)$. In fact, if $l_{0}$ is minimal with this property, then $X=X_{1} \oplus X_{2}$ is an $\left(l_{0}-1\right)$ division of the $\Lambda$-module $X$.

Since we assumed that $\operatorname{Ext}_{\Lambda / \mathfrak{A}}^{d+1}\left(X_{1},-\right) \simeq \operatorname{Ext}_{\Lambda / \mathfrak{A}}^{d+1}\left(X_{2},-\right)$ then, using Lemma 4.1, we obtain that $\operatorname{Ext}_{\Lambda}^{d+r+1}\left(X_{1},-\right) \simeq \operatorname{Ext}_{\Lambda}^{d+r+1}\left(X_{2},-\right)$.

Hence, $l=d+r+1>d$ satisfies $\operatorname{Ext}_{\Lambda}^{l}\left(X_{1},-\right) \simeq \operatorname{Ext}_{\Lambda}^{l}\left(X_{2},-\right)$. So we found $l$ as required, proving that $\phi_{l}^{\Lambda}(X) \geq d$. This proves (a), and (b) follows immediately.

We observe that $\phi_{l}$ can be replaced by $\phi_{r}$ in the previous theorem, since $\phi_{r}(X)=\phi_{l}(D X)$.

These results apply to any convex subcategory $\Delta$ of a quiver algebra $\Lambda=$ $k Q / I$, where $Q$ is a finite quiver and $I$ is an admissible ideal of the path algebra $k Q$. That is, $\Delta=k Q^{\prime} /\left(k Q^{\prime} \cap I\right)$, where $Q^{\prime}$ is a full convex subquiver of $Q$. In this case $\Delta=\Lambda / \mathfrak{A}$, where $\mathfrak{A}$ is the trace of the projective module $P=\bigoplus_{i \notin Q_{0}^{\prime}} P_{i}$. In this situation it is known that $\mathfrak{A}$ is a strong idempotent ideal (9], Ch. II, Lemma 3.7) and we obtain the following corollary.

Corollary 4.4. Let $\Delta$ be a full convex subcategory of the quiver algebra $\Lambda$, and let $\mathfrak{A}$ be the idempotent ideal such that $\Delta=\Lambda / \mathfrak{A}$. If $\mathfrak{A}$ has finite projective dimension then $\phi_{l} \operatorname{dim} \Delta \leq \phi_{l} \operatorname{dim} \Lambda$.

Now we turn our attention to $\Gamma$-modules. We study the behaviour of both Igusa-Todorov functions $\phi$ and $\psi$ under the functor $\operatorname{Hom}_{\Lambda}(P,-): \bmod \Lambda \rightarrow$ $\bmod \Gamma$ restricted to the subcategories $\mathbb{P}_{\infty}$ and $\mathbb{I}_{\infty}$ of $\bmod \Lambda$.

Proposition 4.5. For a $\Lambda$-module $Y \in \mathbb{P}_{\infty}$ the following properties hold:

(a) there exists a d-division of $Y$ if and only if there exists a d-division of $\operatorname{Hom}_{\Lambda}(P, Y)$.

(b) $\phi_{l}^{\Lambda}(Y)=\phi_{l}^{\Gamma}\left(\operatorname{Hom}_{\Lambda}(P, Y)\right)$.

(c) $\psi_{l}^{\Lambda}(Y)=\psi_{l}^{\Gamma}\left(\operatorname{Hom}_{\Lambda}(P, Y)\right)$.

Proof. (a) Since $\operatorname{Hom}_{\Lambda}(P,-): \bmod \Lambda \rightarrow \bmod \Gamma$ induces an equivalence of categories $\mathbb{P}_{1} \rightarrow \bmod \Gamma$ and $Y \in \mathbb{P}_{\infty} \subseteq \mathbb{P}_{1}$, it follows that $Y=Y_{1} \oplus Y_{2}$ if and only if $\operatorname{Hom}_{\Lambda}(P, Y)=\operatorname{Hom}_{\Lambda}\left(P, Y_{1}\right) \oplus \operatorname{Hom}_{\Lambda}\left(P, Y_{2}\right)$. The statement follows from the fact that $Y_{1}, Y_{2} \in \mathbb{P}_{\infty} \operatorname{implies} \operatorname{Ext}_{\Lambda}^{i}\left(Y_{j},-\right) \simeq \operatorname{Ext}_{\Gamma}^{i}\left(\operatorname{Hom}_{\Lambda}\left(P, Y_{j}\right), \operatorname{Hom}_{\Lambda}(P,-)\right)$, for $j=1,2$ and for all $i \geq 0$ ([1], Theorem 3.2,(c)). 
(b) This is a direct consequence of (a), using that $\phi_{l}^{\Lambda}(Y)=n$ if and only if $n=\max (\{d \in \mathbb{N}$ : there exists a $d$-division of $Y\} \cup 0)$ by Theorem 3.6 in [5].

(c) Let $X \in \bmod \Gamma$. Then $\psi_{l}^{\Gamma}(X)=n+l$, with $n=\phi_{l}^{\Gamma}(X)$ and $l=\operatorname{pd} Z_{1}$, where $Z_{1}$ is the largest summand of $\Omega^{n}(X)$ of finite projective dimension. We write $\Omega^{n}(X)=Z_{1} \oplus Z_{2}$.

Let $Y \in \mathbb{P}_{\infty} \subseteq \mathbb{P}_{1}$ be such that $X=\operatorname{Hom}_{\Lambda}(P, Y)$. Since $\phi_{l}^{\Lambda}(Y)=\phi_{l}^{\Gamma}(X)$, we only need to prove that $l$ is the projective dimension of largest summand $Y_{1}$ of $\Omega^{n}(Y)$ of finite projective dimension. Let $\Omega^{n}(Y)=Y_{1} \oplus Y_{2}$ and let

$$
\cdots P_{n+1} \rightarrow P_{n} \rightarrow \cdots \rightarrow P_{0} \rightarrow Y \rightarrow 0
$$

be a minimal projective resolution of $Y$ in $\bmod \Lambda$. Since $Y \in \mathbb{P}_{\infty}$ then

$$
\cdots \rightarrow \operatorname{Hom}_{\Lambda}\left(P, P_{n}\right) \rightarrow \cdots \rightarrow \operatorname{Hom}_{\Lambda}\left(P, P_{0}\right) \rightarrow \operatorname{Hom}_{\Lambda}(P, Y) \rightarrow 0
$$

is a minimal projective resolution of $X=\operatorname{Hom}_{\Lambda}(P, Y)$ in $\bmod \Gamma$, as follows from [1, Lemma 3.1.

Therefore $\Omega^{n}(X) \simeq \operatorname{Hom}_{\Lambda}\left(P, \Omega^{n}(Y)\right)$. This is, $Z_{1} \oplus Z_{2} \simeq \operatorname{Hom}_{\Lambda}\left(P, Y_{1}\right) \oplus$ $\operatorname{Hom}_{\Lambda}\left(P, Y_{2}\right)$. Since $Y \in \mathbb{P}_{\infty}$, then $\Omega^{n}(Y)$ and all direct summands of $\Omega^{n}(Y)$ are also in $\mathbb{P}_{\infty}$. Thus $\operatorname{pd} L=\operatorname{pdHom}_{\Lambda}(P, L)$ for any summand $\mathrm{L}$ of $\Omega^{n}(Y)$ (see 1], Corollary 3.3). From this we conclude that the projective dimensions of the largest summands of finite projective dimension of $\Omega^{n}(X)$ and $\Omega^{n}(Y)$ coincide, as desired.

We state the corresponding result for the $\phi$-injective dimension in the next proposition.

Proposition 4.6. For a $\Lambda$-module $Y \in \mathbb{I}_{\infty}$ the following properties hold:

(a) there exists a d-division of $Y$ if and only if there exists a d-division of $\operatorname{Hom}_{\Lambda}(P, Y)$.

(b) $\phi_{r}^{\Lambda}(Y)=\phi_{r}^{\Gamma}\left(\operatorname{Hom}_{\Lambda}(P, Y)\right)$.

(c) $\psi_{r}^{\Lambda}(Y)=\psi_{r}^{\Gamma}\left(\operatorname{Hom}_{\Lambda}(P, Y)\right)$.

Proof. The result follows using that $\operatorname{Hom}_{\Lambda}(P,-): \mathbb{I}_{1} \rightarrow \bmod \Gamma$ is an equivalence of categories, the fact that $\operatorname{Ext}_{\Lambda}^{i}(-, Y) \simeq \operatorname{Ext}_{\Gamma}^{i}\left(\operatorname{Hom}_{\Lambda}(P,-), \operatorname{Hom}_{\Lambda}(P, Y)\right)$ for all $Y \in \mathbb{I}_{\infty}$ and $i \geq 0$ ([1], Theorem 3.2, (b)), and dualizing arguments in the proof of the previous proposition.

Since $\operatorname{Hom}_{\Lambda}(P,-): \bmod \Lambda \rightarrow \bmod \Gamma$ induces equivalences of categories $\mathbb{P}_{1} \rightarrow \bmod \Gamma$ and $\mathbb{I}_{1} \rightarrow \bmod \Gamma$, the previous propositions yield the following result.

Theorem 4.7. (a) If $\mathbb{P}_{1}=\mathbb{P}_{\infty}$ then $\phi_{l} \operatorname{dim} \Gamma \leq \phi_{l} \operatorname{dim} \Lambda$ and $\psi_{l} \operatorname{dim} \Gamma \leq$ $\psi_{l} \operatorname{dim} \Lambda$.

(b) If $\mathbb{I}_{1}=\mathbb{I}_{\infty}$ then $\phi_{r} \operatorname{dim} \Gamma \leq \phi_{r} \operatorname{dim} \Lambda$ and $\psi_{l} \operatorname{dim} \Gamma \leq \psi_{l} \operatorname{dim} \Lambda$.

Our next objective is to find bounds for the $\phi$ dimension of $\Lambda$ in terms of the $\phi$ dimensions of $\Lambda / \mathfrak{A}$ and $\Gamma$. 
We start with the case when the global dimension of $\Gamma$ is finite. Let $\mathbb{T}$ be the subcategory of $\bmod \Lambda$ considered in the previous section, consisting of the modules $T$ such that $\operatorname{Ext}_{\Lambda}^{i}(\Lambda / \mathfrak{A}, T)=0$ for all $i \geq 1$. We observe first that bounds for the function $\phi$ in the subcategory $\mathbb{T}$ will give us bounds for $\phi$ in $\bmod \Lambda$, as the following lemma shows.

Lemma 4.8. Let $\mathfrak{A}$ be an idempotent ideal. Then

(a) $\phi_{r} \operatorname{dim}(\Lambda) \leq \operatorname{pd}_{\Lambda}(\Lambda / \mathfrak{A})+\phi_{r} \operatorname{dim}(\mathbb{T})$

(b) $\phi_{l} \operatorname{dim}(\Lambda) \leq \operatorname{pd}(\Lambda / \mathfrak{A})_{\Lambda}+\phi_{l} \operatorname{dim}(\tilde{\mathbb{T}})$.

Proof. If $\operatorname{pd}_{\Lambda}(\Lambda / \mathfrak{A})=\infty$ there is nothing to prove. Assume $\operatorname{pd}_{\Lambda}(\Lambda / \mathfrak{A})=t<$ $\infty$ and let $X \in \bmod \Lambda$. Then by Lemma 5.5 in $\left[1, \Omega^{-t}(X) \in \mathbb{T}\right.$. The lemma follows now by repeated use of the dual of the inequality of Lemma 1.3 in 7, $\phi_{r}(X) \leq t+\phi_{r}\left(\Omega^{-t}(X)\right.$. This proves (a), and (b) follows by duality.

Observe now that when $\mathfrak{A}$ is a strong idempotent ideal then $\mathfrak{A}$ is in $\mathbb{P}_{\infty}([1]$, Theorem 2.1'), so $\operatorname{pd}_{\Lambda}(\mathfrak{A}) \leq \operatorname{pd}_{\Gamma}\left(\operatorname{Hom}_{\Lambda}(P, \mathfrak{A})\right) \leq \operatorname{gld} \Gamma$. Thus $\operatorname{pd}_{\Lambda}(\Lambda / \mathfrak{A}) \leq$ gld $\Gamma+1$. Since being a strong idempotent ideal is a symmetric condition we obtain that $\operatorname{pd}(\Lambda / \mathfrak{A})_{\Lambda} \leq \operatorname{gld} \Gamma+1$, as observed in [1] at the end of section 5 .

Proposition 4.9. Let $\mathfrak{A}$ be a strong idempotent ideal. Then

$$
\phi_{r} \operatorname{dim}(\mathbb{T}) \leq \max \left\{\operatorname{gld}(\Gamma)+1, \phi_{\mathrm{r}} \operatorname{dim}(\Lambda / \mathfrak{A})+\operatorname{pd}(\Lambda / \mathfrak{A})_{\Lambda}\right\} .
$$

Proof. Let now $r=\operatorname{pd}(\Lambda / \mathfrak{A})_{\Lambda}, T$ in $\mathbb{T}$ and consider the glueing sequence $0 \rightarrow \tau_{\Lambda / \mathfrak{A}} T \rightarrow T \rightarrow T / \tau_{\Lambda / \mathfrak{A}} T \rightarrow 0$ in $\mathbb{T}$. Since $T / \tau_{\Lambda / \mathfrak{A}} T$ is in $\mathbb{I}_{\infty}$, we know by [1, Corollary $3.3 \mathrm{~b}$ ) that injdim $\operatorname{dim}_{\Lambda} T / \tau_{\Lambda / \mathfrak{A}} T=\operatorname{injdim}_{\Gamma} \operatorname{Hom}_{\Lambda}\left(P, T / \tau_{\Lambda / \mathfrak{A}} T\right)$. Then, the corresponding long exact sequence of functors yields isomorphisms of functors $\delta_{i}: \operatorname{Ext}_{\Lambda}^{i}\left(-, \tau_{\Lambda / \mathfrak{A}} T\right) \rightarrow \operatorname{Ext}_{\Lambda}^{i}(-, T)$ for $i>\operatorname{gld} \Gamma+1$.

It is enough to show that $\phi_{r}^{\Lambda}(T) \leq \phi_{r}^{\Lambda / \mathfrak{A}}\left(\tau_{\Lambda / \mathfrak{A}} T\right)+r$, whenever $\phi_{r}^{\Lambda}(T)>$ $\operatorname{gld} \Gamma+1$. With this purpose we assume that $d=\phi_{r}^{\Lambda}(T)>\operatorname{gld} \Gamma+1$ and that $T=T_{1} \oplus T_{2}$ is a $d$-injective-division in $\bmod \Lambda$. We start by proving that $\tau_{\Lambda / \mathfrak{A}} T=\tau_{\Lambda / \mathfrak{A}} T_{1} \oplus \tau_{\Lambda / \mathfrak{A}} T_{2}$ is a j-injective division in $\bmod \Lambda / \mathfrak{A}$, for some $j$ such that $d+1 \leq j+r$. Since $T=T_{1} \oplus T_{2}$ is a $d$-injective-division of $T$, then $\operatorname{Ext}_{\Lambda}^{d}\left(-, T_{1}\right) \not \operatorname{Ext}_{\Lambda}^{d}\left(-, T_{2}\right)$ and $\operatorname{Ext}_{\Lambda}^{d+1}\left(-, T_{1}\right) \simeq \operatorname{Ext}_{\Lambda}^{d+1}\left(-, T_{2}\right)$. Therefore, since $T_{1}$ and $T_{2}$ are also in $\mathbb{T}$ we have that $\operatorname{Ext}_{\Lambda}^{d}\left(-, \tau_{\Lambda / \mathfrak{A}} T_{1}\right) \not \subset \operatorname{Ext}_{\Lambda}^{d}\left(-, \tau_{\Lambda / \mathfrak{A}} T_{2}\right)$ and $\operatorname{Ext}_{\Lambda}^{d+1}\left(-, \tau_{\Lambda / \mathfrak{A}} T_{1}\right) \simeq \operatorname{Ext}_{\Lambda}^{d+1}\left(-, \tau_{\Lambda / \mathfrak{A}} T_{2}\right)$ in $\bmod \Lambda$, by the isomorphisms above.

Now, since $\mathfrak{A}$ is a strong idempotent ideal we deduce from the last isomorphism that $\operatorname{Ext}_{\Lambda / \mathfrak{A}}^{d+1}\left(-, \tau_{\Lambda / \mathfrak{A}} T_{1}\right) \simeq \operatorname{Ext}_{\Lambda / \mathfrak{A}}^{d+1}\left(-, \tau_{\Lambda / \mathfrak{A}} T_{2}\right) \operatorname{in} \bmod \Lambda / \mathfrak{A}$.

On the other hand, $\operatorname{pd}(\Lambda / \mathfrak{A})_{\Lambda} \leq \operatorname{gld} \Gamma+1$ as we observed just before the statement of the proposition. Since we assumed that $\operatorname{gld}(\Gamma)+1<d$ we obtain that $\operatorname{pd}(\Lambda / \mathfrak{A})_{\Lambda}+1=r+1 \leq d$. We conclude then, from Lemma 4.2 that $\operatorname{Ext}_{\Lambda / \mathfrak{A}}^{d-r}\left(-, \tau_{\Lambda / \mathfrak{A}} T_{1}\right) \not \operatorname{Ext}_{\Lambda / \mathfrak{A}}^{d-r}\left(-, \tau_{\Lambda / \mathfrak{A}} T_{2}\right)$ in $\bmod \Lambda / \mathfrak{A}$. This fact and the isomorphism $\operatorname{Ext}_{\Lambda / \mathfrak{A}}^{d+1}\left(-, \tau_{\Lambda / \mathfrak{A}} T_{1}\right) \simeq \operatorname{Ext}_{\Lambda / \mathfrak{A}}^{d+1}\left(-, \tau_{\Lambda / \mathfrak{A}} T_{2}\right)$ obtained above imply 
that $\tau_{\Lambda / \mathfrak{A}} T=\tau_{\Lambda / \mathfrak{A}} T_{1} \oplus \tau_{\Lambda / \mathfrak{A}} T_{2}$ is a j-injective division in $\bmod \Lambda / \mathfrak{A}$, for some $j$ such that $d-r \leq j \leq d$. Thus $d \leq j+r \leq \phi_{r} \operatorname{dim} \Lambda / \mathfrak{A}+r$. This proves that $\phi_{r}(T) \leq \phi_{r} \operatorname{dim} \Lambda / \mathfrak{A}+r$, provided gld $\Gamma+1>d=\phi_{r}(T)$, and ends the proof of the proposition.

Proposition 4.10. Let $\mathfrak{A}$ be a strong idempotent ideal. Then

$$
\phi_{r} \operatorname{dim}(\Lambda) \leq \operatorname{pd}_{\Lambda}(\Lambda / \mathfrak{A})+\max \left\{\operatorname{gld}(\Gamma)+1, \operatorname{pd}(\Lambda / \mathfrak{A})_{\Lambda}+\phi_{r} \operatorname{dim}(\Lambda / \mathfrak{A})\right\} .
$$

Proof. The result follows from Lemma 4.8 and Proposition 4.9 .

Corollary 4.11. Let $\mathfrak{A}$ be a strong idempotent ideal. Assume $\operatorname{gld}(\Gamma)<\infty$, then $\phi_{r} \operatorname{dim}(\Lambda / \mathfrak{A})$ is finite if and only if $\phi_{r} \operatorname{dim}(\Lambda)$ is finite.

Since convex subalgebras of $\Lambda$ are obtained as factors of $\Lambda$ by a strong idempotent ideal, the previous results apply to them. In particular, we obtain the following corollary.

Corollary 4.12. Let $\Delta$ be a full convex subalgebra of $\Lambda$, and let $\mathfrak{A}=\tau_{P}(\Lambda)$ be the idempotent ideal such that $\Delta=\Lambda / \mathfrak{A}$. If $\Gamma$ has finite global dimension, then $\phi_{r} \operatorname{dim}(\Delta)$ is finite if and only if $\phi_{r} \operatorname{dim}(\Lambda)$ is finite.

Example 4.13. Let $\Lambda=\left(\begin{array}{cc}A & 0 \\ M & B\end{array}\right)$, where $A$ and $B$ are artin algebras, $\operatorname{gld}(B)<\infty$ and $M$ is a $B$-A-bimodule. Then $\phi_{r} \operatorname{dim}(\Lambda)$ is finite if and only if $\phi_{r} \operatorname{dim}(A)$ is finite.

In particular we obtain that a one point co-extension of $A$ has finite $\phi_{r} \operatorname{dim}$ if and only if $A$ does.

Next we illustrate the previous Proposition with the following example.

Example 4.14. Let $\Lambda$ be the algebra given by the quiver $Q$

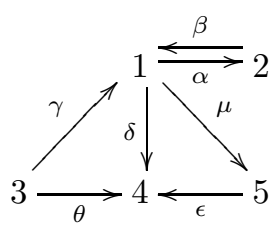

with relations $\alpha \beta=\beta \alpha=0, \mu \gamma=0, \delta \gamma=0, \epsilon \mu=0$. Let $P=P_{3} \oplus P_{4} \oplus P_{5}$, and let $\mathfrak{A}=\tau_{P}(\Lambda)$. Then $\mathfrak{A} \simeq P_{3} \oplus P_{4}^{3} \oplus P_{5} \oplus S_{5}^{2}$, so $\mathrm{pd} \mathfrak{A}=1$. Moreover, since there is an exact sequence $0 \rightarrow P_{4} \rightarrow P_{5} \rightarrow S_{5} \rightarrow 0$ we obtain that $S_{5} \in \mathbb{P}_{\infty}$, so $\mathfrak{A} \in \mathbb{P}_{\infty}$ and is thus a strong idempotent. Then the quiver of $\Lambda / \mathfrak{A}$ is

$$
1 \stackrel{\beta}{\stackrel{\beta}{\rightleftarrows}} 2
$$


with radical square zero, so $\Lambda / \mathfrak{A}$ is selfinjective and therefore $\phi_{r} \operatorname{dim} \Lambda / \mathfrak{A}=0$, by [6. On the other hand $\Gamma$ is the hereditary algebra with quiver

$$
3 \underset{\theta}{\longrightarrow} 4 \leftarrow \epsilon
$$

and we get

$$
\phi_{r} \operatorname{dim}(\Lambda) \leq 2+\max \{1+1,2+1\}=5 .
$$

We now turn our attention to the case when $\operatorname{gld}(\Lambda / \mathfrak{A})$ is finite.

Proposition 4.15. Let $\mathfrak{A}$ be a strong idempotent ideal and assume that $\operatorname{gld}(\Lambda / \mathfrak{A})$ is finite. Then

(a) $\phi_{r}(T) \leq \max \left\{\operatorname{gld}(\Lambda / \mathfrak{A})+\operatorname{pd}(\Lambda / \mathfrak{A})_{\Lambda}+1, \phi_{\mathrm{r}} \operatorname{dim}\left(\mathrm{T} / \tau_{\Lambda / \mathfrak{A}} \mathrm{T}\right)\right\}$ for any $T \in \bmod \Lambda$.

(b) $\phi_{r} \operatorname{dim}(\mathbb{T}) \leq \max \left\{\operatorname{gld}(\Lambda / \mathfrak{A})+\operatorname{pd}(\Lambda / \mathfrak{A})_{\Lambda}+1, \phi_{r} \operatorname{dim}(\Gamma)\right\}$.

Proof. Let $s=\operatorname{gld}(\Lambda / \mathfrak{A}), r=\operatorname{pd}(\Lambda / \mathfrak{A})_{\Lambda}$ and let $X$ be a $\Lambda / \mathfrak{A}$-module.

We claim that $\operatorname{Ext}_{\Lambda}^{i}(-, X)=0$ for all $i \geq s+r+1$. In fact, since gld $\Lambda / \mathfrak{A}=s$ we know that $\operatorname{Ext}_{\Lambda / \mathfrak{A}}^{j}(-, X)=0$ for all $j \geq s+1$. Then $\operatorname{Ext}_{\Lambda}^{j+r}(-, X)=0$ by Lemma 4.2 , for $j \geq s+1$. So the claim holds.

Let now $T$ in $\bmod \Lambda$ and consider the sequence $0 \rightarrow \tau_{\Lambda / \mathfrak{A}} T \rightarrow T \rightarrow$ $T / \tau_{\Lambda / \mathfrak{A}} T \rightarrow 0$. The corresponding long exact sequence of functors yields an isomorphism of functors $\delta_{i}: \operatorname{Ext}_{\Lambda}^{i}(-, T) \rightarrow \operatorname{Ext}_{\Lambda}^{i}\left(-, T / \tau_{\Lambda / \mathfrak{A}} T\right)$ for each $i \geq s+r+1$.

We prove next that a $d$-division of $T$ in $\bmod \Lambda$ yields a $d$-division $T / \tau_{\Lambda / \mathfrak{A}} T$ in $\bmod \Lambda$.

In fact, let $d=\phi_{r} \operatorname{dim}(T)$ and let $T=T_{1} \oplus T_{2}$ be a $d$-division of $T$. This is, $\operatorname{Ext}_{\Lambda}^{d}\left(-, T_{1}\right) \not \operatorname{Ext}_{\Lambda}^{d}\left(-, T_{2}\right)$ and $\operatorname{Ext}_{\Lambda}^{d+1}\left(-, T_{1}\right) \simeq \operatorname{Ext}_{\Lambda}^{d+1}\left(-, T_{2}\right)$. Assume now $d \geq s+r+1$. The functorial isomorphisms $\operatorname{Ext}_{\Lambda}^{i}\left(-, T_{k}\right) \simeq$ $\operatorname{Ext}_{\Lambda}^{i}\left(-, T_{k} / \tau_{\Lambda / \mathfrak{A}} T_{k}\right), k=1,2$ and $i \geq s+r+1$ induced by $\delta_{i}$ show that $T / \tau_{\Lambda / \mathfrak{A}} T=T_{1} / \tau_{\Lambda / \mathfrak{A}} T_{1} \oplus T_{2} / \tau_{\Lambda / \mathfrak{A}} T_{2}$ is a $d$-division of $T / \tau_{\Lambda / \mathfrak{A}} T$ in $\bmod \Lambda$. Therefore, $\phi_{l}^{\Lambda}\left(T / \tau_{\Lambda / \mathfrak{A}} T\right) \geq d$. This ends the proof of $(a)$.

Assume finally that $T \in \mathbb{T}$. Therefore $T / \tau_{\Lambda / \mathfrak{A}} T \in \mathbb{I}_{\infty}$. We know by Proposition 4.6 (b) that $\phi_{r}^{\Gamma} \operatorname{Hom}_{\Lambda}\left(P, T / \tau_{\Lambda / \mathfrak{A}} T\right) \geq d$. This proves that $\phi_{r} \operatorname{dim} \mathbb{T} \leq$ $\max \left\{s+r+1, \phi_{r} \operatorname{dim} \Gamma\right\}$.

Proposition 4.16. Let $\mathfrak{A}$ be a strong idempotent ideal. Then $\phi_{r} \operatorname{dim}(\Lambda) \leq$ $\operatorname{pd}_{\Lambda} \Lambda / \mathfrak{A}+\max \left\{\operatorname{gld}(\Lambda / \mathfrak{A})+\operatorname{pd}(\Lambda / \mathfrak{A})_{\Lambda}+1, \phi_{r} \operatorname{dim}(\Gamma)\right\}$

Proof. If $\operatorname{gld} \Lambda / \mathfrak{A}=\infty$ there is nothing to prove. If gld $\Lambda / \mathfrak{A}$ is finite, the result follows from the previous proposition and Lemma 4.8

Next we obtain another bound for $\phi_{l} \operatorname{dim}(\Lambda)$ with different methods. 
Lemma 4.17. Let $\mathfrak{A}$ be a strong idempotent ideal. Let $0 \rightarrow X \rightarrow Y \rightarrow Z \rightarrow 0$ be an exact sequence in $\bmod \Lambda$ with $X \in \mathbb{P}_{\infty}$ and $Z \in \bmod \Lambda / \mathfrak{A}$ such that $\operatorname{pd}_{\Lambda / \mathfrak{A}} Z$ is finite. Then

(a) $\Omega^{n}(Y) \in \mathbb{P}_{\infty}$ for $n \geq \operatorname{pd}_{\Lambda / \mathfrak{A}} Z$.

(b) $\phi_{l}^{\Lambda}(Y) \leq \operatorname{pd}_{\Lambda / \mathfrak{A}} Z+\phi_{l} \operatorname{dim} \Gamma$.

Proof. (a) Let $\cdots P_{n} \rightarrow \cdots \rightarrow P_{2} \rightarrow P_{1} \rightarrow P_{0} \rightarrow X \rightarrow 0$ and $\cdots Q_{n} \rightarrow \cdots \rightarrow$ $Q_{2} \rightarrow Q_{1} \rightarrow Q_{0} \rightarrow Z \rightarrow 0$ be minimal projective resolutions in $\bmod \Lambda$.

Since $\mathfrak{A}$ is a strong idempotent ideal then $\cdots Q_{n} / \mathfrak{A} Q_{n} \rightarrow \cdots \rightarrow Q_{2} / \mathfrak{A} Q_{2} \rightarrow$ $Q_{1} / \mathfrak{A} Q_{1} \rightarrow Q_{0} / \mathfrak{A} Q_{0} \rightarrow Z \rightarrow 0$ is a minimal projective resolution of $Z$ in $\bmod \Lambda / \mathfrak{A}$ (Theorem 1.6 iii) in [1]). Then $Q_{n} / \mathfrak{A} Q_{n}=0$ for $n>s=\operatorname{pd}_{\Lambda / \mathfrak{A}} Z$. So $Q_{n}=\mathfrak{A} Q_{n}=\tau_{P} Q_{n}$ is in add $P$ for $n>s$ and therefore $\Omega^{n}(Z) \in \mathbb{P}_{\infty}$ for $n>s$.

On the other hand, we assumed that $X$ is in $\mathbb{P}_{\infty}$, so that $\Omega^{n}(X)$ is also in $\mathbb{P}_{\infty}$. Let $n>s$. Since $\cdots P_{r} \oplus Q_{r} \rightarrow \cdots \rightarrow P_{1} \oplus Q_{1} \rightarrow P_{0} \oplus Q_{0} \rightarrow Y \rightarrow 0$ is a projective resolution of $Y$ and $P_{r} \oplus Q_{r} \in \operatorname{add} P$ for $r \geq n$, then $\Omega^{n}(Y) \in \mathbb{P}_{\infty}$. This proves (a).

(b) Let $s=\operatorname{pd}_{\Lambda / \mathfrak{A}} Z$. By a) we know that $\Omega^{n}(Y)$ is in $\mathbb{P}_{\infty}$, for all $n \geq s$. Thus $\phi_{l}^{\Lambda}(Y) \leq \phi_{l}^{\Lambda}\left(\Omega^{s}(Y)\right)+s=\phi_{l}^{\Gamma}\left(\operatorname{Hom}_{\Lambda}\left(P, \Omega^{s}(Y)\right)\right)+s \leq \phi_{l} \operatorname{dim} \Gamma+s$, where the first inequality is given by Lemma 1.3 in [7, and the equality follows from Proposition 4.5 (b).

Proposition 4.18. Let $\mathfrak{A}$ be a strong idempotent ideal. Then

(a) $\phi_{l} \operatorname{dim}(\tilde{\mathbb{T}}) \leq \operatorname{gld}(\Lambda / \mathfrak{A})+\phi_{l} \operatorname{dim} \Gamma$.

(b) $\phi_{l} \operatorname{dim}(\Lambda) \leq \operatorname{pd}(\Lambda / \mathfrak{A})_{\Lambda}+\operatorname{gld}(\Lambda / \mathfrak{A})+\phi_{l} \operatorname{dim}(\Gamma)$.

Proof. (a) Taking supremum on $T \in \tilde{\mathbb{T}}$ and using (b) of the previous lemma applied to the glueing sequence $0 \rightarrow \tau_{\mathfrak{A}} T \rightarrow T \rightarrow T / \tau_{\mathfrak{A}} T \rightarrow 0$, we get $\phi_{l} \operatorname{dim}(\tilde{\mathbb{T}}) \leq \phi_{l} \operatorname{dim} \Gamma+\operatorname{gld}(\Lambda / \mathfrak{A})$.

(b) If $\operatorname{gld} \Lambda / \mathfrak{A}=\infty$ there is nothing to prove. If $\operatorname{gld} \Lambda / \mathfrak{A}$ is finite, by Lemma 4.8(b), we get that $\phi_{l} \operatorname{dim}(\Lambda) \leq \operatorname{pd}(\Lambda / \mathfrak{A})_{\Lambda}+\phi_{l} \operatorname{dim}(\tilde{\mathbb{T}}) \leq \operatorname{pd}(\Lambda / \mathfrak{A})_{\Lambda}+$ $\operatorname{gld}(\Lambda / \mathfrak{A})+\phi_{l} \operatorname{dim}(\Gamma)$

\section{REFERENCES}

[1] M. Auslander, M. I. Platzeck, G. Todorov. Homological theory of idempotent ideals. Trans Am. Math. Soc., vol. 332, n. 2, 667-692, (1992).

[2] A. Beligiannis, I. Reiten. Homological and homotopical aspects of torsion theories. Mem. Amer. Math. Soc., vol. 188, n. 883, viii+207 pp., (2007).

[3] H. Cartan, S. Eilenberg. Homological algebra. Princeton Landmarks in Mathematics. Princeton University Press, xvi+390 pp., (1999).

[4] S. E. Dickson. A torsion theory for abelian categories. Trans. Amer. Math. Soc. 121, 223-235, (1966).

[5] S. Fernandes, M. Lanzilotta, O. Mendoza. The $\phi$-dimension: a new homological measure. Algebras and Representation Theory vol. 18,(2), 463-476, (2015). 
[6] F. Huard, M. Lanzilotta, Self-injective right artinian rings and Igusa-Todorov functions, Algebras and Representation Theory, 16 (3), pp. 765-770, (2012).

[7] F. Huard, M. Lanzilotta, O. Mendoza. An approach to the Finitistic Dimension Conjecture. J. of Algebra 319, 3918-3934, (2008).

[8] K. Igusa, G. Todorov. On the finitistic global dimension for artin algebras. Representation of algebras and related topics, Fields Institute Communications 45 (American Mathematical Society, Providence, RI), 201-204, (2005).

[9] S. Michelena. Sobre un problema de clasificación y cohomología de Hochschild de extensiones locales, Tesis Doctor en Matemática, Universidad Nacional del Sur, (1998).

[10] D. Xu. Homological dimensions and strongly idempotent ideals. J. Algebra 414, 175189, (2014).

María Andrea Gatica:

Instituto de Matemática de Bahía Blanca,

Universidad Nacional del Sur,

Av. Alem 1253, B8000CPB,

Bahía Blanca, ARGENTINA.

mariaandrea.gatica@gmail.com

Marcelo Lanzilotta:

Instituto de Matemática y Estadística Rafael Laguardia (IMERL),

Universidad de la República.

J. Herrera y Reissig 565 C.P. 11300, Montevideo, URUGUAY.

marclan@fing. edu.uy

María Inés Platzeck:

Instituto de Matemática de Bahía Blanca,

Universidad Nacional del Sur,

Av. Alem 1253, B8000CPB,

Bahía Blanca, ARGENTINA.

platzeck@uns.edu.ar 